\title{
A novel sensor for detection of methanol vapor based on conductive amorphous polymer composite: carbon black/poly(etherimide)
}

\author{
Souvik Bag ${ }^{1}$ and Kaushik $\mathrm{Pal}^{1,2^{*}}$ \\ ${ }^{1}$ Centre of Nanotechnology, and ${ }^{2}$ Department of Mechanical and Industrial Engineering, \\ Indian Institute of Technology Roorkee, Roorkee -247667, India. \\ *Email:pl_kshk@yahoo.co.in
}

In rapid progress in $R \& D$ laboratories, industrial productions (semiconductor, petroleum, and chemical, etc.), volatile organic compounds (VOCs) are extensively used during experiment. They sometimes act as reagents or raw materials for making new chemicals and materials. Among of the VOCs, Methanol makes much more attention due to toxicity in nature $[1,2]$. We have developed a sensitive composite based chemiresistive sensor using poly (etherimide) and carbon black for the detection of methanol vapor at room temperature in the range of 1 to 15 partsper-million (ppm). The experimental results indicate that relatively less mass fraction ( $2 \mathrm{wt} \%)$ of carbon black is associated with the composites. When the composite is exposed to methanol $\left(\mathrm{CH}_{3} \mathrm{OH}\right)$ vapors, the electric resistance of the composite drastically increases over a period of time at different concentration level (as shown in Figure 1).

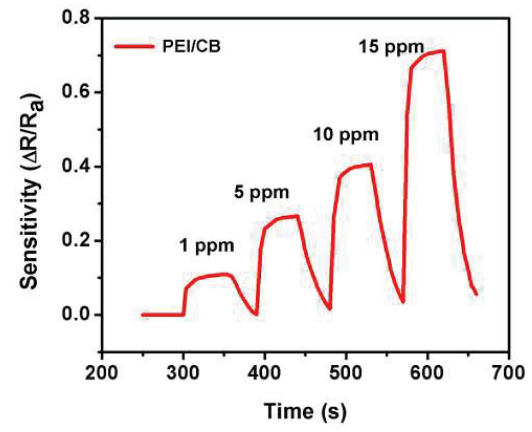

Figure 1: Sensitivity of the PEI/CB based Sensor at various concentration of methanol vapor (1 ppm, 5 ppm, 10 ppm, 15 ppm)

Thus, the sensor displayed highest sensitivity at 15 ppm of methanol vapor. Having high porosity, the composite shows a significant adsorption of methanol vapors on the entire surface of the composite film (as shown in Figure 2). In case of thermal stability, composite showed a significant stability in air atmosphere (as shown in Figure 3).

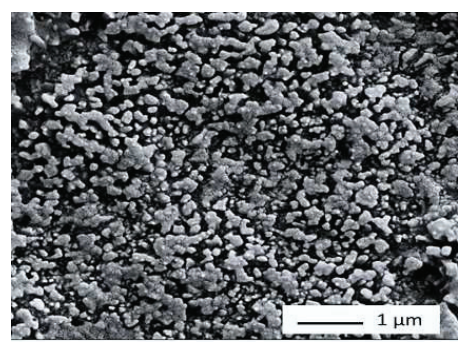

Figure 2: FESEM images of sensing film based on $\mathrm{PEI} / \mathrm{CB}$ composite

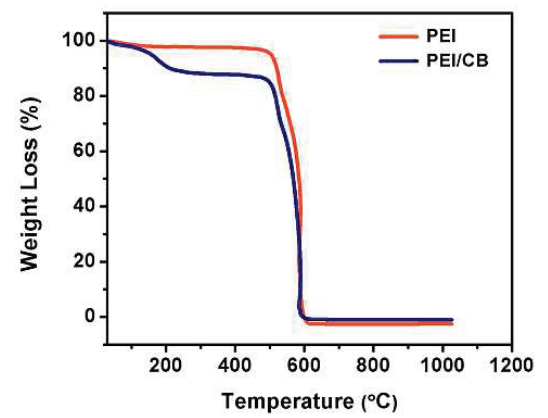

Figure 3: TGA of the pure PEI and PEI/CB based composite in air atmosphere at a ramp of $5{ }^{\circ} \mathrm{C} / \mathrm{min}$

The room temperature operation under ambient conditions indicates that, the composite can be explored for health as well as environment monitoring applications. In addition, the polymer composite based sensor depicted good repeatability as well as selectivity with fast response. The simplicity and low fabrication cost of the resistive sensor with ambient working temperature pave the way for its easy detection of methanol vapor.

\section{References}

1. C.L. Yuana, C.P. Chang, Y. Song, Materials Science and Engineering B 176 (2011) 821-829.

2. H.C. Liao, C.P. Hsu, M.C. Wu, C.F. Lu, and W.F. Su, Anal. Chem. 85 (2013) 9305-9311. 